\title{
XIX.
}

Ueber die Zusammensetzung des in den schwedischen Gebirgen vorkommenden Feldspaths und über die Bestandtheile des Hälleflinta's.

\author{
Von
}

\section{F. Svanberg.}

(Förhandl. vid de Skand. Naturf. IIIge Möte; i Stockholm, Juli 1842.)

Ungeachtet man seit längerer Zeit hinsichtlich der Kenntniss der die sogenannten primitiven oder massigen Gebirgsarten constituirenden Gemengtheile im Klaren zu sein glaubt, so ist mir doch nicht bekannt, auf welchen speciellen Untersuchungen diese Kenntniss beruht, und ich vermuthe, dass die Bestandtheile dieser Gebirgsarten ursprünglich öfter auf einen ganz losen Grund hin für das angesehen worden sind, wofür man sie später gehalten hat, als dass die Bestimmung derselben auf gründlichen Untersuchungen beruhte. So sieht man Quarz, Glimmer und Feldspath als die Hauptbestandtheile des Granits an, ohne dass diese Mineralien im Granite näher bestimmt und untersucht worden wären. Dessenungeachtet hat man gefunden, dass, auch bei einem sich ziemlich gleichbleibenden Korne und Farbe, dennoch Granite von verschiedenem Aussehen und geologischem Verhalten vorgekommen sind. Die Folge hiervon ist auch gewesen, dass der schwedische Granit von ausländischen Geologen zuweilen ganz von der Granitreihe verwiesen und dem Gneisse zugerechnet, zuweilen wiederum von dem Gneisse verwiesen und als zum Granit gehörig betrachtet worden ist, weswegen denn in spätern Zeiten der höchst eigenthümliche Name Granit-Gneiss demselben beigelegt worden ist. Die Unbestimmtheit des Begriffes von Granit und Gneiss ist hiervon die Ursache.

Als ich einige hierher gehörige Mineralien chemisch genau untersuchte, hat sich ergeben, dass das feldspathartige Mineral in mehreren der schwedischen Urberge nicht Orthoklas sei (wenn man nämlich darunter die chemische Verbindung versteht, welche durch die mineralogische Formel $K S_{3}+3 A S_{3}$ ausgedrückt wird), sondern dass theils Oligoklas, theils durchaus neue, mit demselben sowohl in nuturhistorischer wie in chemischer Hinsicht 
verwandte Mineralien in diesen Felsarten als constituirende Bestandtheile vorhanden sind. Es ist klar, dass die nähere Untersuchung dieser Bestandtheile für die Kenntniss der verschiedenen Granitarten überhaupt von grosser Wichtigkeit sein muss; denn gleichwie die Versteinerungen die geologischen Hauptcharaktere darbieten, um die geschichteten Gebirgsarten zu bestimmen und zu unterscheiden, eben so müssen für die massigen Felsarten die constituirenden Bestandtheile ähnliche Merkmale zur Unterscheidung der verschiedenen Arten derselben liefern, d. h. die Mineralien sind für die massigen Gebirgsarten durchaus dasselbe, wie die Petrefacten für die geschichteten. Wohl kann man es als wahrscheinlicb betrachten, dass in den verschiedenen Epochen der Bildung von massigen Felsarten sich auch verschiedene Verwandtschaften zwischen den Elementen geltend gemacht haben, auf die Art nämlich, dass die Silicate, in welchen die Kieselsäure z. B. 3 mal so viel Sauerstoff enthält als die Basen, in einer andern Periode entstanden sind, als wenn dieselbe nur 2 mal den Sauerstoff der Basen in sich aufgenommen hat; dass ferner die Doppelsilicate, in welchen die Thonerde 3 mal so viel Sauerstoff enthält als die Alkalien, durch andere Umstände hervorgerufen worden sind, als wenn das Verhältniss zwischen diesen Sauerstofmengen $=2: 1$ ist; dass Kali, Natron und Kalkerde unter bestimmt verschiedenen Momenten auftreten; dass auch die Amphibole mit und ohne Thonerde und Eisen ein durchaus andres Verhalten voraussetzen; alle diese wie noch mehrere andere hiermit im Zusammenhange stehende Fragen sind bis jetzt ihrer Lösung noch keineswegs entgegengeführt worden.

Die Untersuchungen, welche in dieser Hinsicht gegenwärtig in Schweden angestellt werden, sind erst in ihrer Kindheit; aber da die schwedische Bergwerksgesellschaft (Svenska Brukssocietet) mit gewöhnlichem Eifer diese für unseres Landes geognostische Kenntniss wichtige Frage aufgefasst, so wie auch die nöthigen Geldmittel für die jährlichen, in dieser Hinsicht anzustellenden Forschungen mitgetheilt hat, so ist zu vermuthen, dass wir über diesen Gegenstand mit der Zeit uns eine vollkommnere Kenntniss werden verschaffen können. Was mich betrift, so ist mir nur die chemische Untersuchung der besonders charakteriatisch verschiedenen Arten, die von den eigentlichen Geologen gesammelt worden sind, anfgetragen worden, und icl werde 
schwed. Gebirgen vorkommenden Feldspaths etc. 163

hier nur im Allgemeinen die Zusammensetzung einiger der Feldspatharten mittheilen, welche bei diesen geognostischen Forschungen bis jetzt sind angetroffen worden.

Das feldspathartige Mineral von Berga in Vestra Wingåkers Kirchspiel in Södermannland zeigte sich zusammengesetzt $=2 r$ $S_{4}+3 A S_{4}$, worin $r$, welches 14,8 Proc. beträgt, aus $6,1 \mathrm{Kali}$, 5,2 Natron und 3,5 Kalkerde besteht.

Das Mineral von Magsjö und Tanså besteht aus $r S_{3}+2 A S_{3}$, und das Magsjö-Mineral enthält in hundert Theilen 9,8 Kali und 3,3 Natron, während das Tansa-Mineral 10,9 Proc. Kali und 3,6 Natron enthält.

Die Mineralien von Bredsjö und Tomtebo sind Orthoklase $r S_{3}+3 A S_{3}$ und enthalten als substituirenden Theil in geringer Quantitüt das in diesem Mineral oft vorkommende Natron. So enthält der Feldspath von Bredsjö in Procenten 11,1 Kali und 2,2 Natron, der von Tomtebo 10,5 Kali und 2,8 Natron.

Dit 0elsjö- und Wedevägs-Mineralien bestehen aus $r S_{3}+$ 3 A $S_{2}$; das erstere enthäl in hundert Theilen $0,9 \mathrm{Kali}, 8,7 \mathrm{Na}$ tron, 3,3 Kalkerde, und das Ietztere von Wedevăg $7,5 \mathrm{Kali}$, 3,1 Natron und 3,4 Kalkerde.

Das feldspathartige Mineral in Rapakivi von Aborforss in Finnland ist zusammengesetzt nach der Formel $2 \tau S_{3}+5 A S_{2}$, in welcher die einatomigen Basen bestehen aus 10,2 Kali, 3,0 Natron und 4,7 Kalkerde.

Die Mineralien also von Berga, Magsjö, Tanså und Aborforss bieten Verbindungen dar, nach Typen, wie sie bis jetzt noch nicht beobachtet worden sind. Das Mineral von Wedevåg ist wohl auch eine neue Species, aber gehört za demselben Genus wie der Oligoklas, dessen Natron hier durch Kali ersetzt worden ist. Alle diese Mineralien, mit Ausnahme des ron Oelsjö, welches ein reiner Oligoklas ist, enthalten zu gleicher Zeit sowohl Kali als Natron. Die Mittheilung der ansführlichen Details der Zusammensetzung dieser Mineralien schiebe ich auf, bis eine grössere Mlenge der hierber gehörigen Fossilien untersucht worden ist.

Schliesslich will ich noch Einiges über den Hälleflinta mittheilen. Dieses Mineral tritt theils gangförmig in andern Bergen, theils auch als selbstständige Felsart auf. Seine vielfachen Namen, wie Petrosilex, Eurit, Hornfels, Trapp, Leptunit und 
164 Sranberg: Zusammensetzung des Feldspaths etc.

Porphyr, mit allen ihren Varietäten, zeigen schon hinlänglich, dass man über diese Bergart der Hauptsache nach noch sehr in Ungewissheit ist. So hat man diese Gebirgsart zuweilen als aus dichtem Feldspath bestehend betrachtet, während man auf der andern Seite geglaubt hat, dieselbe bestehe allein aus Quarz, mit einer unberleutenden Einmengung von Feldspath. Etwas völlig Entscheidendes kann auch auf chemischem Wege nicht erreicht werden, da dieses Fossil, in Folge seiner unkrystallinischen Textur, wahrscheinlich oft nur aus verschiedenen zusammengeschmolzenen Mineralmassen besteht. Wie auch die Bildung desselben mag gewesen sein, so ergiebt sich aus seiner Zusammensetzung, dass oft ganz verschiedene Quantitiaten Kieselerde in demselben enthalten sind, weswegen mun auch, wenn die Zusammensetzung des Hïlleflinta's auf einer einzelnen Stelle gleichförmig ist, die Analyse als den Ausdruck des Verhälnisses zwischen den Total-Bestandiheilen der Gebirgsart ansehen kann, auf die Art, als ob man eine Analyse zum Beispiel von irgend einem bestimmten massigen Granite ausgefiihrt hätte. Ohne deswegen den Hälleflint für cine einfache mineralogische Verbindung zu halten, will ich hier die mineralogischen Formeln anführen, welchen sich derselbe auf den verschiedenen Stellen in seiner Zusammensetzung zu nühern scheint, wobei ich zugleich bemerke, dass das berechnete Resultat für irgend einen Bestandtheil niemals um 1 Procent von dem gefundenen abweicht.

Hellrother Hälleflinta von Persberg in Wermland war zusammengesetzt nach der Formel $r S_{8}+3 \mathrm{~A} \mathrm{~S}_{4}$, worin $r$ in Procenten aus 0,1 Kali, 5,9 Natron und 1,2 Kalkerde besteht.

Der etwas dunkler rothe Hälleflint, ebenfalls von Persberg in Wermland, bestand aus $2 r S_{6}+5 A S_{4}$ mit 0,5 Kali, 6,5 Natron und 0,5 Kalkerde in Procenten.

Die Porphyrmasse von Gustafström besteht aus $r S_{6}+4 A S_{6}$. $r$ enthält 3,6 Kali, 2,1 Natron und 0,8 Proc. Kalkerde.

Der Hälleflint von Saxå in Helleforss Kirchspiel in Westmannland besteht aus $r S_{j}+3 A S_{6}$, worin $r=2,4$ Proc. Kali, 3,6 Natron und 2,5 Kieselerde.

Der Dannemora-Hällellint, welcher auf dieser Stelle den Namen Dannemora-Bandjaspis erhalten hat, besteht aus dunkleren und helleren Streifen. Beide Arten yon Schichten enthalten bedeutende Einmengung en von kohlensaurem Kalk, und die hellen 
noch mehr als die dunklen. So enthalten die dusklen Sclichten 15\%/3 Proc. kohlensauren Kalk, neben einem Silicat, welches nach der Formel $2 r S_{3}+3 A S_{2}$ zusammengesetzt ist und worin $r$ in den übrigen $84 \frac{1}{3}$ Procenten aus $6,0 \mathrm{Kali}, 1,7$ Natron und 8,0 Kalkerde besteht. Dagegen enthalten die hellen Streifen beinahe 26 Proc. kohlensauren Kalk, vermischt mit einem Silicat, welches nach der mineralogischen Formel $r S_{3}+A S_{2}$ zusammengesetzt ist und worin $r$ in den übrigen $7 \pm$ Proc. 3,2 Kali, 0,1 Natron und 8,1 Kalkerde umfasst.

Der Hälleflint von Sala ist $\boldsymbol{r} \boldsymbol{S}_{2}+\mathbf{3} \boldsymbol{A} \boldsymbol{S}_{2}$, mit nur einer Spur von Kali, 7,1 Proc. Natron und 7,9 Kalkerde.

Der Hälleflint von Stampers Hof in der Nähe von Sala besteht aus $2 r S_{2}+5 A S_{2}$, worin $r 0,4$ Proc. Kali, 6,4 Natron und 6,1 Kalkerde enthält.

Der schwarze Hälleflint vom Hellefors-Stollen zeichnet sich vor allen übrigen durch seine tief schwarze Farbe aus. Derselbe unterscheidet sich auch durch seine Zusammensetzung, da er sowohl Wasser, wie auch Eisenoxydul und Talkerde enthiilt. Seine Zusammensetzung ist jedoch von der Art, dass dieselbe sich auf keine Weise durch irgend eine mineralogische Formel darstellen lässt, da die Sauerstoffmengen im Wasser, den einatomigen Basen, der Thonerde und der líeselerde, sich verhalten $=$ $3: 4: 5: 38$.

\section{XX.}

Ueber einige neue nordische Mineralien.

\section{von \\ A. Erdmann.}

(Förhdl. vid de Skandin. Naturf. IIIge Möte. 1842.)

Herr Erdma nn zeigte ein ueues Mineral, den Bamlit, vor, welches in der Umgegend von Brewig in Norwegen gefunden worden ist. Es kommt daselbst in der Nähe des Hofes Bräkke im Kirchspiel Bamle in dem dort herrschenden Gneisse vor, in welchem es theils in derben strahligen Massen und theils, obgleich selten, krystallisirt in schmalen kleinen, plattgedrückten Prismen eingewachsen ist, welche an den Enden schief abge- 